\title{
BMJ Open Correlation between biomarkers of pain in saliva and PAINAD scale in elderly people with cognitive impairment and inability to communicate: descriptive study protocol
}

\author{
Vanesa Cantón-Habas, ${ }^{1}$ María del Pilar Carrera-González (iD , ${ }^{1}$ \\ María Teresa Moreno-Casbas, ${ }^{2,3}$ José Manuel Quesada-Gómez, ${ }^{4}$ \\ Manuel Rich-Ruiz ${ }^{1,3}$
}

To cite: Cantón-Habas V, Carrera-González MdP, MorenoCasbas MT, et al. Correlation between biomarkers of pain in saliva and PAINAD scale in elderly people with cognitive impairment and inability to communicate: descriptive study protocol. BMJ Open 2019;9:e032927. doi:10.1136/ bmjopen-2019-032927

- Prepublication history for this paper is available online To view these files, please visit the journal online (http://dx.doi org/10.1136/bmjopen-2019032927).

Received 15 July 2019 Revised 15 October 2019 Accepted 16 0ctober 2019

Check for updates

(c) Author(s) (or their employer(s)) 2019. Re-use permitted under CC BY-NC. No commercial re-use. See rights and permissions. Published by BMJ.

For numbered affiliations see end of article.

Correspondence to Dr María del Pilar CarreraGonzález; pcarrera@uco.es

\section{ABSTRACT}

Introduction Pain is an under-diagnosed problem in elderly people, especially in those with cognitive impairment who are unable to verbalise their pain. Although the Pain assessment in advanced dementia scale (PAINAD) scale is a tool recognised for its clinical interest in this type of patients, its correlation with the saliva biomarkers reinforced its utility. The aim of this research will be to correlate the scores of this scale with the levels of biomarkers of pain found in saliva samples of patients with cognitive impairment and inability to communicate. Methods and analysis This is an observational study. The level of pain will be evaluated using the PAINAD scale. Moreover, pain biomarkers, in particular secretory IgA and soluble tumour necrosis factor receptor type II, will be determined in saliva. Both assessments will be conducted in 75 patients aged over 65 years with advanced cognitive impairment and inability to communicate. The PAINAD scores will be correlated with the levels of these biomarkers of pain. A control group consisting of 75 healthy subjects aged over 65 years will be included in the study. Moreover, sociodemographic variables and variables related to pain, dementia and other clinical conditions will be recorded. The analysis will be performed with the statistical package SPSS V.22 and the software R.

Ethics and dissemination The study has been reviewed and approved by the Andalusian Human Research Ethics Committee. In addition, this study has been financed by the Junta de Andalucía through a regional health research fund (Research code: PI-0357-2017). The results will be actively disseminated trough a high-impact journal in our study area, conference presentations and social media.

\section{INTRODUCTION}

The assessment of pain in elderly people with cognitive impairment is often insufficient and, consequently, pain treatment is inadequate. ${ }^{1-4}$

Although elderly patients with cognitive impairment are not the only underdiagnosed and undertreated group, they are undoubtedly one of the most affected. The reasons are mainly two:
Strengths and limitations of this study

- The study will provide a reliable and reinforced tool (the PAINAD scale) to assess pain in elderly people with cognitive impairment and inability to communicate which will facilitate the correct pain management.

- The determination of biomarkers of pain in saliva is a promising tool because it is a noninvasive and inexpensive method that provides information and complements the information provided by the PAINAD scale.

- The results of the present study will lead to an improvement in quality of life of these patients and their families thanks to a better diagnosis of pain using the PAINAD scale and the salivary biomarkers.

- The correlation of the information provided by the PAINAD scale and the values of the salivary biomarkers will allow health institutions to make clinical recommendations with a higher level of evidence.

1. Elders are more likely to experience pain. ${ }^{5}$

2. Elders usually have difficulty communicating the level of pain they are suffering to their caregivers and healthcare providers. $^{36-8}$

Pain is a frequent experience for many elderly people. ${ }^{5}$ Most common chronic diseases afflicting the elderly, such as depression, cardiovascular disease, cancer and osteoporosis, involve a larger risk of developing chronic pain. ${ }^{49}$ Approximately $50 \%$ of community dwelling adults and up to $80 \%$ of institutionalised older adults are estimated to experience considerable pain. ${ }^{710}$

In addition, pain has important consequences in the elderly population, because it affects them both physically and psychologically and, often, pain can lead to dependence 
situations. Pain causes long-term problems affecting, in general, to quality of life. ${ }^{6}$

However, despite being a frequent and important problem, studies have shown that pain is often not evaluated or treated correctly, especially in the elderly.

Numerous studies have also shown that older people with cognitive impairment, particularly with dementia, often suffer from painful illnesses (specifically, it is estimated that between $80 \%$ and $85 \%$ of them suffer pain) and that they are usually prescribed fewer analgesics than patients with intact cognitive abilities. ${ }^{2} 311$

This undertreatment is undoubtedly related to the difficulty of detecting pain in this population, mainly due to the loss of verbal abilities to express pain, ${ }^{3}$ or to insufficient instruction or training of professionals and caregivers to identify pain. ${ }^{12}$

The tools used to assess pain can be classified into: (1) self-reports of pain; (2) direct observation of the person's behaviour and (3) biomarkers.

Self-reports are considered the most reliable and refined measure of the presence and intensity of pain, even in patients with moderate dementia. ${ }^{13}$ However, when the capacity for abstract reasoning is low, the use of such scales, even though they are very simple, becomes very complicated, because patients do not understand the concepts used. This complexity becomes impossible to use when, in addition, the ability of verbal communication is affected.

As an alternative to verbal evaluation (or self-report), a significant number of observational scales have been developed in the last 15 years. Several literature review studies describe more than 24 tools of this type ${ }^{14-21}$ and although none of the tools can be recommended based on existing evidence, several studies advocate the inclusion of any of them within a comprehensive pain care protocol. ${ }^{1022-25}$

Among them, the PAINAD scale is recommended by the National Nursing Home Pain Collaborative as a clinically useful tool, ${ }^{26}$ and several authors describe the PAINAD as the most practical and promising scale. ${ }^{81927-30}$ The PAINAD scale has a convergent validity and a moderate internal consistency. ${ }^{31}{ }^{32}$ The scores obtained with the PAINAD scale vary when performing a potentially painful activity; ${ }^{32} 33$ and the scores decrease after the administration of analgesics. ${ }^{2}$ Despite this, the validation of the PAINAD scale to Spanish is not complete.

The determination of biomarkers of pain is the third of the potential tools. Their determination in saliva would be an enormously useful, noninvasive and economic tool. In fact, some pain biomarkers have already been determined in saliva, ${ }^{3435}$ such as salivary cortisol, its salivary levels correlate strongly with the level of pain, ${ }^{36}$ salivary $\alpha$-amylase,${ }^{37}$ secretory IgA (sIgA) ${ }^{38}$ testosterone ${ }^{39}$ or tumour necrosis factor receptor type II (sTNF-RII) ${ }^{40}$ The saliva levels of sTNF-RII correlate significantly with its levels in plasma. ${ }^{41}$ However, authors such as Sobas et $a t^{42}$ point to sIgA and sTNF-RII, out of all these biomarkers, as potential salivary markers of pain in healthy people, since they presented the highest intraindividual reproducibility.

\section{METHODS AND ANALYSIS}

\section{Aim and objectives}

The aim of this research will be to correlate the scores of the PAINAD scale with the levels of pain biomarkers in saliva samples obtained from patients with cognitive impairment. The objectives of the study will be to:

1. Evaluate the pain level through the PAINAD scale.

2. Determine in saliva the values of the pain biomarkers sTNF-RII and sIgA in a population with cognitive impairment and communication inability.

3 . Identify the possible relation between sociodemographic and clinical variables and the PAINAD score and the values of pain biomarkers in saliva.

\section{Research hypothesis}

We anticipate that the PAINAD score will correlate with the salivary levels of sTNF-RII and sIgA in the sample. The correlation level will be established between the final score on the scale and the presence of significant differences in these biomarkers.

\section{Study design}

This is an observational study which began in May 2018 and will end in June 2020. The design followed the Strengthening the Reporting of Observational Studies in Epidemiology recommendations.

This study is funded by the Health Department of the Regional Government of Andalusia (PI-0357-2017).

\section{Study setting}

A health district of an Andalusian province, through its network of Primary Healthcare centres and an institution dedicated to the care of patients with dementia, specifically, with Alzheimer's disease.

\section{Participants and selection criteria}

The sample size has been calculated for a correlation magnitude of $\mathrm{r}=0.3$, a statistical CI of $95 \%$, a statistical power of $80 \%$, with a unilateral approach and a $10 \%$ of losses. The result sample size is 75 subjects.

The inclusion criteria for participants were the following:

- Age $\geq 65$ years.

- Medical diagnosis of dementia or Alzheimer's disease with a Global Deterioration Scale (GDS) score between 5 and $7 .^{43}$

- Being unable to communicate verbally.

- Having a relative or legal representative that can sign the informed consent for the participation of the patient in the study.

- Being included, at least, for 3 months in the listings of the dementia process. In the case of the institution dedicated to the care of patients with Alzheimer's disease, patients who have used this service for at least 3 months will be included.

The recruitment of the participants will be conducted consecutively by the interventional nurses belonging to the different participating primary healthcare centres, among the subjects that attend their usual nurse 


\begin{tabular}{ll} 
Table 1 Study measures & \\
\hline Sociodemographic data & Clinical history \\
\hline Health variables & Clinical history \\
Pharmacological treatments & Clinical history \\
Cognitive impairment & GDS \\
Autonomy in activities of daily living & Barthel Index \\
PAINAD & Observational scale \\
Biomarkers & Saliva collection \\
\hline
\end{tabular}

GDS, Global Deterioration Scale.

consultation based on the lists of the dementia process of the urban health centres (HC) and an institution dedicated to the care of Alzheimer's patients.

Subjects without cognitive impairment, within the same age range, who voluntarily wish to participate in this study, will be investigated to correlate the values obtained in the PAINAD with the determination of pain biomarkers. These 75 control subjects will be recruited from the environment close to the subjects (family, relatives and other users of the same $\mathrm{HC}$ ).

\section{Study measures}

The main variables will be the scores of the Spanish version of PAINAD and the determinations of the biomarkers sTNF-RII and IgAs. Other study variables will be the scores of the GDS scale; sociodemographic variables (age and sex); clinical data related to pain (duration, frequency, location, aetiology, type of analgesic treatment, adjuvant treatment and pain control at the current time) and level of autonomy in basic activities of daily living (table 1 ).

\section{Data collection}

A specific Book for Data Collection (BDC) will be provided to each researcher. An explaining manual for the BDC has been developed for standardised data collection to ensure the quality of data collection. In addition, the professionals will receive face-to-face training about data collection and the tools or assessment scales to ensure the validity and reproducibility.

The professionals will also be individually instructed on how to perform the saliva collection using the passive secretion method: ${ }^{44}$

1. One hour prior to sample collection, subjects should not eat, drink (except water), chew gum, brush their teeth, consume caffeine or do physical exercise.

2. Five minutes prior to sample collection, the subject should rinse their mouth with clean water to reduce the contamination of saliva with food debris.

3. All existing saliva in the mouth should be swallowed before starting the sample collection.

4. Subsequently, intermittently deposit the accumulated saliva for a period of $5 \mathrm{~min}$ in a collection tube, requiring at least $1 \mathrm{~mL}$. If the $5 \mathrm{~mL}$ collection tube is filled before $5 \mathrm{~min}$, the amount of time that has elapsed is recorded.
Voluntary written informed consent will be obtained from a family member or their legal representative in the case of patients with cognitive impairment, and directly from the control group subjects. Once the consent is received, the researcher will collect the sociodemographic and clinical data of the patient. The PAINAD scale will be completed along with the other selected measures. In addition, saliva will be collected.

Samples will be collected in a clinical setting, under supervision, between 09:00 and 10:00 in the morning and always before the morning medication is taken. The samples collected will always be refrigerated, and will be collected always in the same room, where temperature and humidity will be recorded. After collection, the samples will be frozen at $-80^{\circ} \mathrm{C}$ until analysis.

Data will be collected by qualified personnel previously instructed under the aforementioned protocol.

\section{Determination of sTNF-RII and IgA}

The determination of sTNF-RII and IgAs levels will be performed using an ELISA. The sTNF-RII levels will be determined using the Human sTNF-RII Quantikine ELISA kit (R\&D Systems, Minneapolis, MN) and the IgAs using the sIgA ELISA kit (Salimetrics LLC, State College, Pennsylvania, USA). The collection period will be determined because IgA levels depend on the flow of saliva secretion. The quantification of total proteins in saliva will be performed using Bradford's method, using bovine serum albumin as standard.

\section{Data analysis}

For the description of the sample, the number of observations, mean, $\mathrm{SD}$, minimum, maximum, median, IQR and 95\% CI for the mean value will be used for quantitative variables; whereas the absolute and relative frequencies will be used for categorical variables (for this type of variables, the lost data will appear as another category, with its absolute frequency and its percentage).

In order to determine the degree of relationship between the PAINAD scale and the values of the biomarkers considered, the matrix of polyserial correlations with their corresponding hypothesis contrasts will be used. A logit model will be used to determine which biomarker most affects the pain condition and multinomial logistic models will be considered to determine which of the two biomarkers has the strongest influence on pain gradation.

Next, to determine the possible relationships between demographic and clinical variables with PAINAD scores and biomarkers, Pearson and polyserial correlations will be obtained depending on the scales considered (continuous and ordinal), as well as the $\chi^{2}$ coefficient or predictive coefficients lambda (nominal and ordinal), with their corresponding significances levels. Finally, in the case of detecting some type of relationship between these variables, they will be introduced in the logit and logistic multinomial models to determine their relative influence on the appearance and degree of pain. 
This analysis will be carried out using the statistical package SPSS V.22 for most calculations and the software R for the determination of the coefficients of polyserial correlations. For all cases, a significance of $5 \%$ will be assumed.

\section{Patient and public involvement}

The research question for this study was developed based on a synthesis of the recent literature, therefore patients and the public were not involved in the design of the study, including the research question, outcomes measures, recruitment to or conduct of the study. The results of the study will be actively disseminated and made available to the heads of the participating centres.

\section{DISCUSSION}

The severe ageing of global population has caused a significant increase in the prevalence of cognitive impairment. However, the importance of these figures is not due solely to the current transcendence of this public health problem, but also it is due to the fact that multiple studies indicate that the percentage of people with dementia will increase substantially in the coming years. ${ }^{45}$

In this context, healthcare systems, particularly in Western countries, face new challenges in the care of patients with cognitive impairment, among them the assessment and adequate management of pain are significant, needing solutions to provide quality care to this population.

Pain in patients with cognitive impairment is an unresolved problem due to the progression of the neurological disorders that they present and that entails the loss of the ability to communicate, which makes it difficult to manage pain and, therefore, it leads to pain underdiagnosis and undertreatment. ${ }^{12}$

The PAINAD scale is a tool of acknowledged utility for the assessment of pain in people with cognitive impairment and communication inability. ${ }^{46}$

The determination of pain biomarkers in saliva is a tool of maximum utility because of its non-invasiveness and improved accessibility to the biological sample in comparison with blood determinations, becoming a rigorous and accurate assessment tool thanks to which patient's pain can be detected and addressed.

The correlation of the results of both tools will allow health professionals, as well as relatives and informal caregivers of these patients, to know that pain can be evaluated correctly, allowing a better use of analgesia.

\section{Limitations}

The selection of the subjects studied may be affected by consecutive sampling. However, no previous list of patients, not even an estimate of the total number of patients meeting the established inclusion criteria, was available for the studied location.

In addition, the recruitment process may be affected by the non-acceptance of those relatives of patients who do not want to participate in the study.
Finally, it is necessary to point out that the test-retest has the limitation of the changing nature of pain. Although the study is designed for the chronic pain condition, it is inevitable that cases of acute pain are found and that, therefore, the score changes, even when there is a narrow margin between the two measurements. These cases of acute pain will undoubtedly represent a distortion in the verification of this feature.

\section{ETHICS AND DISSEMINATION}

All participants will be informed of the general aspects of the study. Participants have the right to withdraw consent to participate at any time.

Moreover, all the participants must sign a written informed consent before participating in the study. Confidentiality is guaranteed.

The study will comply with the requirements set out by the Regulation (EU) 2016/679 of the European Parliament and of the Council of 27 April 2016 on General Data Protection Regulation (GDPR).

The plans to disseminate the results of this observational study include publishing at least a research paper in peerreviewed journals in our study area. Also, if possible, the results of this research will be presented in conference presentations.

\section{CONCLUSION}

The results of this research will allow elucidating the concentrations of pain biomarkers in saliva, which would be an objective tool of enormous utility to confirm the possible diagnosis of pain in this population. The correlation between the values of the PAINAD scale and those of the pain biomarkers obtained in saliva samples will reflect the complementarity of both tools for the assessment of pain and will reinforce the suitability of the scale in relation to the evaluation of pain in this population.

\section{Author affiliations}

${ }^{1}$ Instituto Maimónides de Investigación Biomédica (IMIBIC)/ Universidad de Córdoba/ Hospital Universitario Reina Sofía, University of Cordoba, Cordoba, Spain

${ }^{2}$ Nursing and Healthcare Research Unit (Investén-isciii), Institute of Health Carlos III, Ministry of Science and Innovation, Madrid, Spain

${ }^{3}$ Ciber Fragilidad y Envejecimiento Saludable (CIBERFES), Madrid, Spain ${ }^{4}$ Instituto Maimónides de Investigación Biomédica de Córdoba (IMIBIC) \& Unidad de Gestión Clínica (UGC) de Endocrinología y Nutrición, Hospital Universitario Reina Sofía, Córdoba, Spain

Acknowledgements We thank Nutraceutical Translations for the translation of this manuscript into English.

Contributors VC-H, MdPC-G and MR-R conceptualised the project and conceived the study design.VC-H drafted the manuscript. MdPC-G, MR-R, MTM-C and JMQ-G reviewed and edited the draft protocol. All authors read and approved the final manuscript.

Funding This work was supported by the Junta de Andalucía through a regional health research fund (Research code: PI-0357-2017.)

Competing interests None declared.

Patient consent for publication Not required. 
Ethics approval The study will be conducted following the ethical principles of the Helsinki Declaration. In addition, both the design and the development of the work will meet the standards of good clinical practice (CPMP/ICH/135/95, July 2002, European Medicines Agency). The study protocol has been evaluated by a peer-review process and funded by a grant awarded under the call for proposals in Biomedical and Health Sciences $\mathrm{R}+\mathrm{D}+\mathrm{I}$ of the Regional Government of Andalusia (22 December 2017).

Provenance and peer review Not commissioned; externally peer reviewed.

Open access This is an open access article distributed in accordance with the Creative Commons Attribution Non Commercial (CC BY-NC 4.0) license, which permits others to distribute, remix, adapt, build upon this work non-commercially, and license their derivative works on different terms, provided the original work is properly cited, appropriate credit is given, any changes made indicated, and the use is non-commercial. See: http://creativecommons.org/licenses/by-nc/4.0/.

\section{ORCID iD}

María del Pilar Carrera-González http://orcid.org/0000-0001-6575-8240

\section{REFERENCES}

1 Ellis-Smith C, Evans CJ, Bone AE, et al. Measures to assess commonly experienced symptoms for people with dementia in longterm care settings: a systematic review. BMC Med 2016;14:38.

2 Hadjistavropoulos T, Herr K, Prkachin KM, et al. Pain assessment in elderly adults with dementia. Lancet Neurol 2014;13:1216-27.

3 Jensen-Dahm C, Werner MU, Dahl JB, et al. Quantitative sensory testing and pain tolerance in patients with mild to moderate Alzheimer disease compared to healthy control subjects. Pain 2014;155:1439-45.

4 Passmore P, Cunningham E. Pain assessment in cognitive impairment. J Pain Palliat Care Pharmacother 2014;28:305-7.

5 Tracy B, Sean Morrison R. Pain management in older adults. Clin Ther 2013;35:1659-68.

6 Burfield AH, Wan TTH, Sole ML, et al. Behavioral cues to expand a pain model of the cognitively impaired elderly in long-term care. Clin Interv Aging 2012;7:207-23.

7 Kaasalainen S, Akhtar-Danesh N, Hadjistavropoulos T, et al. A comparison between behavioral and verbal report pain assessment tools for use with residents in long term care. Pain Management Nursing 2013;14:e106-14.

8 Lukas A, Schuler M, Fischer TW, et al. Pain and dementia: a diagnostic challenge. Z Gerontol Geriatr 2012;45:45-9.

9 Andrade DC, Faria JWV, Caramelli P, et al. The assessment and management of pain in the demented and non-demented elderly patient. Arq Neuropsiquiatr 2011;69:387-94.

10 Apinis C, Tousignant M, Arcand M, et al. Can adding a standardized observational tool to interdisciplinary evaluation enhance the detection of pain in older adults with cognitive impairments? Pain Med 2014;15:32-41.

11 Bauer U, Pitzer S, Schreier MM, et al. Pain treatment for nursing home residents differs according to cognitive state - a crosssectional study. BMC Geriatr 2016;16:124

12 Lautenbacher S, Niewelt BG, Kunz M. Decoding pain from the facia display of patients with dementia: a comparison of professional and nonprofessional observers. Pain Med 2013;14:469-77.

13 Hadjistavropoulos T, Herr K, Turk DC, et al. An interdisciplinary expert consensus statement on assessment of pain in older persons. Clin J Pain 2007;23:S1-43.

14 Neville C, Ostini R. A psychometric evaluation of three pain rating scales for people with moderate to severe dementia. Pain Manag Nurs 2014;15:798-806.

15 Corbett A, Husebo B, Malcangio M, et al. Assessment and treatment of pain in people with dementia. Nat Rev Neurol 2012;8:264-74.

16 Chow S, Chow R, Lam M, et al. Pain assessment tools for older adults with dementia in long-term care facilities: a systematic review. Neurodegener Dis Manag 2016;6:525-38.

17 Lichtner V, Dowding D, Esterhuizen P, et al. Pain assessment for people with dementia: a systematic review of systematic reviews of pain assessment tools. BMC Geriatr 2014;14:138.

18 Carezzato NL, Valera GG, Vale FAC, et al. Instruments for assessing pain in persons with severe dementia. Dement Neuropsychol 2014;8:99-106

19 Husebo BS, Achterberg W, Flo E. Identifying and managing pain in people with Alzheimer's disease and other types of dementia: a systematic review. CNS Drugs 2016;30:481-97.

20 Bullock L, Bedson J, Jordan JL, et al. Pain assessment and pain treatment for community-dwelling people with dementia: a systematic review and narrative synthesis. Int J Geriatr Psychiatry 2019;34:807-21.

21 Herr K, Zwakhalen S, Swafford K. Observation of pain in dementia. Curr Alzheimer Res 2017;14:486-500.

22 Booker SQ, Haedtke C. Assessing pain in nonverbal older adults. Nursing 2016;46:66-9.

23 Chen $\mathrm{Y}-\mathrm{H}$, Lin L-C. Ability of the pain recognition and treatment (PRT) protocol to reduce expressions of pain among institutionalized residents with dementia: a cluster randomized controlled trial. Pain Manag Nurs 2016;17:14-24.

24 Liu JYW, Lai C. Implementation of observational pain management protocol to improve pain management for long-term institutionalized older care residents with dementia: study protocol for a clusterrandomized controlled trial. Trials 2014;15:78.

25 van der Steen JT, Sampson EL, Van den Block L, et al. Tools to assess pain or lack of comfort in dementia: a content analysis. J Pain Symptom Manage 2015;50:659-75.

26 Herr K, Bursch $\mathrm{H}$, Ersek M, et al. Use of pain-behavioral assessment tools in the nursing home: expert consensus recommendations for practice. J Gerontol Nurs 2010;36:18-29.

27 Fry M, Arendts G, Chenoweth L. Emergency nurses' evaluation of observational pain assessment tools for older people with cognitive impairment. J Clin Nurs 2017;26:1281-90.

28 Ngu SSC, Tan MP, Subramanian P, et al. Pain assessment using self-reported, Nurse-reported, and observational pain assessment tools among older individuals with cognitive impairment. Pain Management Nursing 2015;16:595-601 https://doi.org/

29 Paulson CM, Monroe T, Mion LC. Pain assessment in hospitalized older adults with dementia and delirium. J Gerontol Nurs 2014;40:10-15

30 Somes J, Donatelli NS. Pain assessment in the cognitively impaired or demented older adult. J Emerg Nurs 2013;39:164-7.

31 Paulson-Conger M, Leske J, Maidl C, et al. Comparison of two pain assessment tools in nonverbal critical care patients. Pain Manag Nurs 2011;12:218-24.

32 Lints-Martindale AC, Hadjistavropoulos T, Lix LM, et al. A comparative investigation of observational pain assessment tools for older adults with dementia. Clin J Pain 2012;28:226-37.

33 Jordan A, Hughes J, Pakresi M, et al. The utility of PAINAD in assessing pain in a UK population with severe dementia. Int $J$ Geriatr Psychiatry 2011;26:118-26.

34 Bell RF, SivertsenA, Mowinkel P, et al. A bilateral clinical model for the study of acute and chronic pain after breast-reduction surgery. Acta Anaesthesiol Scand 2001;45:576-82.

35 Singla NK, Desjardins PJ, Chang PD. A comparison of the clinical and experimental characteristics of four acute surgical pain models: dental extraction, bunionectomy, joint replacement, and soft tissue surgery. Pain 2014;155:441-56.

36 McLean SA, Williams DA, Harris RE, et al. Momentary relationship between cortisol secretion and symptoms in patients with fibromyalgia. Arthritis Rheum 2005;52:3660-9.

37 Robles TF, Sharma R, Park K-S, et al. Utility of a salivary biosensor for objective assessment of surgery-related stress. J Oral Maxillofac Surg 2012;70:2256-63.

38 da Silva Campos MJ, Souza Alves CC, Barbosa Raposo NR, et al. Influence of salivary secretory immunoglobulin A level on the pain experienced by orthodontic patients. Med Sci Monit 2010;16:CR405-9.

39 Choi JC, Chung MI, Lee YD. Modulation of pain sensation by stressrelated testosterone and cortisol. Anaesthesia 2012;67:1146-51.

40 Goodin BR, Quinn NB, King CD, et al. Salivary cortisol and soluble tumor necrosis factor- $\alpha$ receptor II responses to multiple experimental modalities of acute pain. Psychophysiology 2012;49:118-27.

41 Nishanian P, Aziz N, Chung J, et al. Oral fluids as an alternative to serum for measurement of markers of immune activation. Clin Diagn Lab Immunol 1998;5:507-12.

42 Sobas EM, Reinoso R, Cuadrado-Asensio R, et al. Reliability of potential pain biomarkers in the saliva of healthy subjects: interindividual differences and Intersession variability. PLoS One 2016;11:e0166976.

43 Reisberg B, Ferris SH, de León MJ, et al. The global deterioration scale for assessment of primary degenerative dementia. Am J Psychiatry 1982;139:1136-9.

44 Golatowski C, Gesell Salazar M, Dhople VM, et al. Comparative evaluation of saliva collection methods for proteome analysis. Clinica Chimica Acta 2013;419:42-6.

45 World Health Organization. Dementia: a public health priority. Ginebra: WHO, 2017.

46 Rababa M. Pain assessment in people with dementia: remaining controversies. Glob J Health Sci 2018;10:62-9. 